Revista Colombiana de Obstetricia y Ginecología Vol. 61 No. 3 • $2010 \bullet(239-246)$

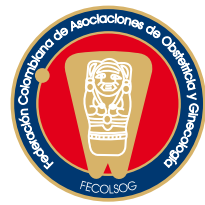

ARTículo de ReVISIÓN

\title{
MANEJO INTEGRAL DE LA GESTANTE CON VIRUS DE LA INMUNODEFICIENCIA HUMANA
}

\section{Integral management of HIV-infected-pregnant femalles}

Camilo Rada-Ortega*, Jorge Mario Gómez-Jiménez, M.D.**

Recibido: noviembre 17/09 - Aceptado: agosto 9/10

\section{RESUMEN}

Objetivo: revisar los aspectos más relevantes del manejo de la gestante con virus de inmunodeficiencia humana (VIH) antes, durante y después del parto. También se incluyen las medidas básicas de cuidado al recién nacido.

Metodología: se realizó una revisión de las publicaciones en MEDLINE entre enero de 2000 y junio de 2009 sobre el tema. Asimismo, se abordaron las guías de manejo del Ministerio de Protección Social de Colombia y las pertenecientes a los Centros para el Control de Enfermedades de los EE.UU. de América.

Resultados: la oportuna prescripción de la terapia antirretroviral de alta efectividad durante el control prenatal, la utilización de profilaxis antirretroviral anteparto, la realización de cesárea anteparto, evitar la lactancia materna y la profilaxis neonatal son las medidas que evitarán casi al 100\% la transmisión madre-hijo del VIH.

Conclusión: el manejo actualmente recomendado permite la prevención de la transmisión madre-hijo del VIH en la mayoría de los casos.

* Estudiante del Nivel XI de Medicina. Facultad de Medicina de la Universidad de Antioquia. Medellín (Colombia). Correo electrónico: drcroe@gmail.com

** Profesor Asociado, Especialista en Obstetricia y Ginecología. Facultad de Medicina de la Universidad de Antioquia. Medellín (Colombia).
Palabras clave: VIH, embarazo, neonato, lactancia, anticoncepción.

\section{SUMMARY}

Objective: reviewing the most relevant aspects involved in managing pregnant females affected by human immunodeficiency virus (HIV) before, during and after giving birth. Basic measures in caring for the newborn are also included.

Methodology: MEDLINE publications on the topic published from January 2000 to June 2009 were reviewed. Colombian Ministry of Social Protection guidelines were also reviewed as well as those from the US Centers for Disease Control and Prevention (CDC).

Results: the opportune prescription of highly effective antiretroviral therapy during prenatal control, using pre-delivery antiretroviral prophylaxis, performing caesarean section, avoiding maternal breastfeeding and neonatal prophylaxis were found to be the measures which would lead to almost $100 \%$ mother-child HIV transmission being avoided.

Conclusion: current management recommendations would lead to preventing mother-child HIV transmission in most cases.

Key words: HIV, pregnancy, newborn, breastfeeding, contraception. 


\section{INTRODUCCIÓN}

La disponibilidad de medicamentos antirretrovirales ha permitido una reducción del porcentaje de niños nacidos de madre VIH positiva, con transmisión del VIH madre-hijo en Europa y Estados Unidos. Este tipo de infección se redujo de una frecuencia del 25\% a menos del 2\%. ${ }^{1}$ Este descenso ocurre por el uso de la de profilaxis perinatal específica, ${ }^{2}$ la práctica de la cesárea en sus respectivas indicaciones y el reemplazo de lactancia materna por leche de fórmula segura para el recién nacido. ${ }^{3,4}$

Se estima que alrededor de 38,6 millones de personas están infectadas con VIH en todo el mundo y que 25 millones han fallecido a causa de la infección. ${ }^{5}$ Por otra parte, de un 25\% a un 30\% de las mujeres con VIH en Norteamérica, que reciben tratamiento para la infección, desean estar embarazadas. ${ }^{6,7}$

Sin embargo, la transmisión madre-hijo del virus, es responsable del 70\% de los casos de niños infectados cada año en el mundo y es más probable que ocurra durante el trabajo de parto ${ }^{8}$ Lo anterior constituye un llamado de atención a los profesionales de la salud, encargados del cuidado obstétrico, para que intervengan de forma oportuna en la prevención de la infección en el recién nacido.

Teniendo en cuenta que la atención obstétrica en Colombia es realizada tanto por médicos generales como por especialistas, con la participación de personal de enfermería, y que la infección con VIH debe ser prevenida y reconocida en estadios iniciales, es necesario disponer de herramientas que nos permitan ofrecer la mejor atención posible a la gestante con VIH, una vez se presenta a la primera consulta prenatal.

El abordaje a la paciente embarazada infectada con VIH debe hacerse en el control prenatal, durante el parto, en el período de lactancia materna y en las primeras semanas de vida del recién nacido, pues es durante estos períodos en los que el personal de salud puede intervenir en el cuidado de la paciente y su producto de concepción, para prevenir la infección resultante de la gestación.
Es por esto que el objetivo de esta revisión es presentar la evidencia disponible respecto al curso de la entidad durante el embarazo y acerca de las recomendaciones para la prevención de la enfermedad madre-hijo en los momentos antes mencionados.

\section{METODOLOGÍA}

En la base de datos PubMed/MEDLINE, se realizó una búsqueda de las publicaciones entre enero de 2000 y junio de 2009. Se utilizaron los términos: "virus de inmunodeficiencia humana", "transmisión madre-hijo", "terapia antirretroviral”, "neonato”. Se incluyeron en la búsqueda investigaciones originales y artículos de revisión, metaanálisis, guías de práctica clínica, entre otros. Por otra parte, se revisaron las guías de manejo del Ministerio de Protección Social de Colombia y las de los Centros para el control de Enfermedades de los EE.UU. de América.

\section{Infección con VIH durante el embarazo}

Estudios realizados en Europa y EE.UU. no muestran que el embarazo contribuya a la progresión de la infección, aunque en países en vía de desarrollo sí se observa un aumento de la progresión de la enfermedad y de la probabilidad de muerte de la madre. ${ }^{9,10}$

Con respecto a los efectos de la infección sobre el embarazo, se ha documentado un pequeño aumento en la tasa de abortos, mortalidad perinatal y neonatal, restricción del crecimiento intrauterino y bajo peso al nacer. ${ }^{11}$

Hasta la fecha no se ha descrito un síndrome de malformación relacionado con la infección con VIH, ni tampoco se ha informado aumento en la frecuencia de defectos congénitos relacionados con la infección. ${ }^{12-14}$

\section{Recomendaciones para el manejo de la infección durante el embarazo}

Para el manejo de la paciente gestante infectada, que acude a consulta antes del parto, el Ministerio de la Protección Social de Colombia dispuso una guía para el manejo de VIH/SIDA, ${ }^{15}$ que propone: 
1. Realizar una prueba de tamizaje (ELISA) en toda paciente que acuda a consulta por primera vez, idealmente en el primer trimestre o en la primera consulta del control prenatal. Esta prueba debe ser antecedida por una explicación clara y coherente de la que se debe dejar constancia firmada de la aceptación o rechazo de su realización. Las pacientes que lleguen en trabajo de parto urgente (expulsivo), se les debe realizar una prueba rápida de tamizaje así no tengan tamización previa.

2. La prueba debe repetirse cuando se diagnostiquen infecciones de transmisión sexual durante el embarazo, uso de drogas intravenosas o múltiples compañeros sexuales.

3. En la paciente VIH positivo: se debe hacer tamizaje para otras infecciones de transmisión sexual, hepatitis B y C. Estos exámenes se realizan para diagnosticar oportunamente comportamientos y conductas sexuales de riesgo, tanto para la madre como para el feto.

4. Idealmente, el manejo debe ser multidisciplinario: se debe incluir un médico general, un obstetra, un neonatólogo o en su defecto un pediatra, y personal de enfermería.

5. La carga viral es el factor más importante para la transmisión vertical y está asociada a la transmisión que ocurre con la lactancia materna. Por lo anterior, se debe iniciar y/o continuar la terapia antirretroviral de alta efectividad, con el objetivo de mantener niveles indetectables de carga viral.

Cuando la paciente tiene un diagnóstico confirmado de infección con VIH y no ha recibido tratamiento, éste se debe iniciar en la semana 14 con terapia antirretroviral de alta efectividad y se debe evitar recibir monoterapia o terapia con dos medicamentos. ${ }^{16}$ Generalmente, la terapia antirretroviral de alta efectividad está compuesta por dos inhibidores análogos de nucleósido de la transcriptasa reversa, junto con un inhibidor análogo no-nucleósido de la transcriptasa reversa o un inhibidor de proteasa. Si la madre ya recibía tratamiento, éste debe ser evaluado antes de continuarse, teniendo en cuenta las siguientes recomendaciones generales que fueron publicadas en la guía de la Public Health Service Task Force en abril de 2009 para el manejo de la paciente gestante infectada con VIH: ${ }^{17}$

1. Terapia antirretroviral o profilaxis para todas las pacientes infectadas (independiente de la carga viral o CD4).

2. Zidovudina incluida en todos los regímenes (debido a su acumulación de forma activa en la placenta).

3. Evaluar la resistencia si hay viremia detectable.

4. Evitar efavirenz en el $1^{\text {er }}$ trimestre (potencial teratogénico).

5. No prescribir las combinaciones: zidovudina + estavudina, didanosina + estavudina $o$ didanosina + zalcitabina. Estas combinaciones pueden generar toxicidad hepática y en el sistema nervioso central, acidosis láctica y disminución de la eficacia de ambos, respectivamente.

\section{Recomendaciones para el manejo de la infección durante el parto}

El riesgo de transmisión madre-hijo de VIH es mayor durante el trabajo de parto que en otros instantes. ${ }^{8}$ Ocurre por la presencia de microtransfusiones de sangre durante las contracciones, por la infección ascendente luego de la ruptura de las membranas y a que el feto degluta secreciones contaminadas con el virus; estas son las hipótesis más representativas que explican el alto riesgo. ${ }^{11}$ Debido a lo anterior, el período intraparto debe ser manejado con base en dos estrategias de intervención, una de ellas la terapia farmacológica y la otra la atención del parto mediante cesárea.

En cuanto a la primera estrategia, la recomendación actual consiste en ofrecer zidovudina sola o combinada con otros medicamentos antirretrovirales a todas las gestantes infectadas. ${ }^{17}$ El esquema consiste en una carga inicial de zidovudina de $2 \mathrm{mg} / \mathrm{kg}$ IV administrados en 1 hora, seguido por infusión 
continua de $1 \mathrm{mg} / \mathrm{kg}$ cada hora hasta finalizar el parto. Si se programa cesárea, el esquema debe iniciarse 3 horas antes del procedimiento quirúrgico.

Otros medicamentos antirretrovirales que la madre haya estado consumiendo durante la gestación se pueden continuar por vía oral, excepto la estadovudina, pues esta presenta antagonismo farmacológico con la zidovudina, lo que influiría en el efecto del esquema anteriormente mencionado.

En cuanto a la realización de la operación cesárea electiva programada, la recomendación actual establecida por el Colegio Americano de Obstetras y Ginecólogos es la realización de cesárea electiva programada (definida como cesárea antes del trabajo de parto y ruptura prematura de membranas) a gestantes con VIH que presenten un nivel de ácido ribonucleico (ARN) viral mayor a 1.000 copias $/ \mathrm{ml}$ en el período cercano al momento del parto. ${ }^{18}$ Para las pacientes que presenten un nivel de ARN viral menor a 1.000 copias $/ \mathrm{ml}$, no hay evidencia clara disponible que recomiende la cesárea electiva programada. ${ }^{19}$ Se recomienda practicarla a las 38 semanas de gestación, siendo estas determinadas por el mejor método clínico y ecográfico. Se debe evitar la realización de amniocentesis. ${ }^{20,21}$

En pacientes con VIH también aplica el uso de profilaxis antimicrobiana. ${ }^{22}$ La morbilidad posparto atribuible a infecciones se aumenta potencialmente con la cesárea, tanto en pacientes seropositivas como seronegativas. Se estima que este tipo de complicaciones es cinco a siete veces más común después de practicarse la cesárea, posterior al inicio del trabajo de parto o ruptura de membranas, comparado con el parto vaginal. ${ }^{23,24}$

Finalmente, debe evitarse la coadministración de metilergonovina e inhibidores de proteasa, debido a la exagerada vasoconstricción que se puede generar durante el manejo de la atonía uterina posparto. ${ }^{17}$

\section{Recomendaciones para el manejo de la infección durante el período posparto}

La morbilidad materna en el período posparto suele aumentar, por lo tanto no se recomienda dar de alta a la paciente de forma temprana. ${ }^{11}$
El seguimiento al binomio madre-hijo debe continuar por parte del médico de atención primaria; además, se debe aconsejar a la madre el uso de métodos anticonceptivos. Hay que tener en cuenta que los niveles de estradiol de algunos anticonceptivos hormonales se reducen con la ingesta simultánea de nevirapina, ritonavir, nelfinavir, rifampina y rifabutina, lo cual puede conducir a la disminución en la eficacia de la anticoncepción. ${ }^{25}$ La terapia antirretroviral de alta efectividad debe continuarse cuando esté indicado y la suspensión de los medicamentos antirretrovirales debe decidirse en conjunto con el médico encargado del manejo de la infección. ${ }^{26}$

\section{Recomendaciones sobre la lactancia materna}

La lactancia materna aumenta la tasa de transmisión materno-fetal del VIH en un 14\% en el caso de la madre infectada antes del parto, y en un 30\% en madres infectadas después de éste. Además existe evidencia de transmisión de $4 \%$ por cada 6 meses de lactancia. ${ }^{27}$ Debido a esto, en países industrializados no se recomienda la utilización de lactancia materna para alimentar al recién nacido, ${ }^{28}$ muy a pesar de los posibles beneficios a corto plazo en términos de proteger contra infecciones virales y bacterianas, o a largo plazo, pues el amamantamiento parece intervenir de forma preventiva en la aparición de problemas médicos de la adultez como diabetes, enfermedades cardiovasculares y cáncer, todas estas de alta prevalencia en dichos países. ${ }^{29-32}$

La discusión se da con respecto a los países en vía de desarrollo. A pesar de que la lactancia materna sea responsable de la infección de 300.000 niños al año, ${ }^{33}$ la UNICEF estima que, a nivel mundial, la falta de lactancia materna es responsable de 1,5 millones de muertes infantiles por año. ${ }^{34}$ Por otra parte, la composición de la leche materna aporta el total de nutrientes requeridos por el niño durante los primeros 6 meses de vida, además, constituye una forma económica y segura de alimentación hasta los dos años de edad, refuerza el vínculo madre-hijo y estimula el desarrollo intelectual. ${ }^{35-37}$ Se debe ha- 
cer un cuidadoso balance riesgo vs. beneficio de la lactancia materna, individualizando cada caso con respecto a la posibilidad de trasmisión, la situación socioeconómica y las propiedades nutricionales que ésta aporta al niño.

Hasta la fecha, solamente se ha realizado un estudio clínico controlado aleatorio sobre lactancia materna vs. leche de fórmula. Este estudio realizado en Kenia arrojó un riesgo de transmisión del 16\% en los primeros 24 meses de edad; ${ }^{28}$ sin embargo, se detectaron algunas limitaciones en el diseño del estudio con respecto a la exclusividad y a la adherencia de la lactancia materna.

Actualmente, ya se encuentran descritos algunos factores de riesgo fuertemente implicados en la transmisión del VIH debido a la lactancia materna. ${ }^{38-39}$ Entre ellos se destacan una carga alta de ARN viral, tanto en plasma como en leche materna, progresión clínica o inmunológica de la enfermedad en la madre (bajos niveles de CD4) y patologías mamarias (mastitis, abscesos, pezones fisurados). ${ }^{40}$

\section{Recomendaciones sobre el cuidado del recién nacido}

Todo recién nacido expuesto al VIH debe recibir terapia con antirretrovirales, idealmente iniciada entre 6 y 12 horas posparto, y que abarque 6 semanas de tratamiento con zidovudina. ${ }^{1}$ Se prefiere zidovudina debido a la falta de evidencia de seguridad para el recién nacido con los otros agentes. ${ }^{41}$ La dosis indicada para el recién nacido a término es $2 \mathrm{mg} / \mathrm{kg}$ vía oral cada 6 horas o intravenoso $1,5 \mathrm{mg} / \mathrm{kg}$ cada 6 horas. ${ }^{15}$ Sin embargo, se ha informado sobre efectos negativos de la zidovudina sobre la hematopoyesis y sobre toxicidad mitocondrial con el uso de la terapia antirretroviral de alta efectividad, por lo cual se recomienda realizar en el recién nacido pruebas de función renal, función hepática, glucemia, electrolitos y hemoleucograma con plaquetas. ${ }^{42}$

Se debe realizar reacción en cadena de polimerasa diagnóstica para VIH al día 1, a las 6 semanas, y luego repetirla a las 12 semanas; si todas las anteriores son negativas, y el niño no ha recibido leche materna, podemos informar a los padres que el niño es VIH negativo. ${ }^{43}$ Sin embargo, la desaparición de anticuerpos maternos debe ser confirmada a los 18 meses de edad. La vacunación se hace de forma rutinaria, esperando la confirmación de la negatividad de la infección para aplicar la BCG. ${ }^{44}$

\section{Terapia antirretroviral y embarazo: consideraciones especiales}

Desde el año 1996, en el que se introdujeron en la práctica clínica los medicamentos antirretrovirales, el número de fármacos disponibles y sus posibles combinaciones, continúan incrementándose hoy en día. ${ }^{45}$ Sin embargo y frecuentemente, la evaluación de la eficacia o efectividad de estos nuevos medicamentos no va sustentada por estudios de la mayor calidad metodológica, razón por la cual algunas instituciones y sociedades científicas internacionales han elaborado y actualizado sus propias recomendaciones sobre el empleo de antirretrovirales. ${ }^{46-48}$

Las recomendaciones sobre el tratamiento de mujeres gestantes con VIH se han basado en la premisa de que las terapias con beneficios reconocidos en mujeres no deben ser modificadas durante el embarazo, excepto cuando se hayan documentado efectos adversos en la madre, feto o recién nacido, y estos efectos adversos superen los beneficios. ${ }^{49}$

Un caso para ilustrar sería el uso de los inhibidores de proteasa y el desarrollo de hiperglucemia. Los informes publicados hasta la fecha no han demostrado que se aumentó el riesgo de desarrollo de dicha complicación durante el embarazo. ${ }^{17}$ Lo mismo sucede con respecto al uso de nevirapina y riesgo de falla hepática, debido a que se desconoce si el embarazo aumenta el riesgo de hepatotoxicidad..$^{50,51}$

\section{CONCLUSIONES}

El cuidado de la paciente gestante con virus de inmunodeficiencia humana $(\mathrm{VIH})$ requiere un abordaje integral por parte del equipo de salud tratante, donde la atención debe comenzar desde 
el momento de ingreso de la paciente al sistema, preferiblemente en la etapa preconcepcional o durante el control prenatal, y luego continuar un manejo estricto que abarca la atención del parto, la lactancia materna y los cuidados al recién nacido. Todo lo anterior con el fin de prevenir la transmisión madre-hijo.

Al igual que en todo paciente, se deben mantener las precauciones universales y ofrecer un ambiente de confidencialidad y respeto por la paciente y su familia.

\section{REFERENCIAS}

1. Aberg JA, Kaplan JE, Libman H, Emmanuel P, Anderson JR, Stone VE, et al. Primary care guidelines for the management of persons infected with human immunodeficiency virus: 2009 update by the HIV medicine Association of the Infectious Diseases Society of America. Clin Infect Dis 2009;49:651-81.

2. Connor EM, Sperling RS, Gelber R, Kiselev P, Scott G, O'Sullivan MJ, et al. Reduction of maternal-infant transmission of human immunodeficiency virus type 1 with zidovudine treatment. Pediatric AIDS Clinical Trials Group Protocol 076 Study Group. N Engl J Med 1994;331:1173-80.

3. ACOG committee opinion scheduled cesarean delivery and the prevention of vertical transmission of HIV infection. Number 219, August 1999. Committee on Obstetric Practice. American College of Obstetricians and Gynecologists. Int J Gynaecol Obstet 2001;73:279-81.

4. Centers for Disease Control and Prevention (CDC). Achievements in public health. Reduction in perinatal transmission of HIV infection- United States, 1985-2005. MMWR Morb Mortal Wkly Rep 2006;55:592-7.

5. UNAIDS. 2006 Report on the global AIDS epidemic: a UNAIDS $10^{\text {th }}$ anniversary special edition. Visitado en 2009 Sep 16. Disponible en http://www.unaids. org/en/KnowledgeCentre/HIVData/EpiUpdate/ EpiUtpdArchive/2006/default.asp

6. Chen JL, Philips KA, Kanouse DE, Collins RL, Miu A. Fertility desires and intentions of HIV-positive men and women. Fam Plann Perspect 2001;33:144-52, 165.

7. Ogilvie GS, Palepu A, Remple VP, Maan E, Heath K, MacDonald G, et al. Fertility intentions of women of reproductive age living with $\mathrm{HIV}$ in British Columbia, Canada. AIDS 2007;21:S83-8
8. UNAIDS. Bangkok. 2004 Report on the global AIDS epidemic: $4^{\text {th }}$ global report. Visitado en 2009 Sep 16. Disponible en http://www.unaids.org/bangkok2004/ gar2004_html/GAR2004_00_en.htm.

9. De Cock KM, Fowler MG, Mercier E, de Vincenzi I, Saba J, Hoff E, et al. Prevention of mother-tochild HIV transmission in resource-poor countries: translating research into policy and practice. JAMA 2000;283:1175-82.

10. Saada M, Le Chanadec J, Berrebi A, Bongain A, Delfraissy JF, Mayaux MJ, et al. Pregnancy and progression to AIDS: results of the French prospective cohorts. SEROGEST and SEROCO Study Groups. AIDS 2000;14:2355-60.

11. Penn Z, Dixit, A. Human immunodeficiency virus infection in Pregnancy. Current Obstetrics \& Gynaecology 2006;16:191-8.

12. Brocklehurst P, French R. The association between maternal HIV infection and perinatal outcome: a systematic review of the literature and meta-analysis. Br J Obstet Gynaecol 1998;105:836-48.

13. Stratton P, Tuomala RE, Abboud R, Rodriguez E, Rich $\mathrm{K}$, Pitt J, et al. Obstetric and newborn outcomes in a cohort of HIV-infected pregnant women. A report of the women and infants transmission study. J Acquir Immune Defic Syndr Hum Retrovirol 1999;20:17986.

14. Lorenzi P, Spicher VM, Laubereau B, Hirschel B, Kind C, Rudin C, et al. Antiretroviral therapies in pregnancy: maternal, fetal and neonatal effects. Swiss HIV Cohort Study, the Swiss Collaborative HIV and Pregnancy Study and the Swiss Neonatal HIV Study. AIDS 1998;12:F241-7.

15. Ministerio de Protección Social, Colombia; 2007. Guía para el manejo de VIH/SIDA basada en la evidencia. Visitado en 2009 Sep 16. Disponible en http://www. pos.gov.co/Paginas/guiadeatenciondelvihsida.aspx

16. Betancur J. Infección VIH y embarazo. En: $13^{\circ}$ curso de actualización en obstetricia y ginecología. Facultad de Medicina. Universidad de Antioquia; 2006. p. 8390.

17. AIDSinfo. 2009 Public Health Service Task Force. Recommendations for Use of Antiretroviral Drugs in Pregnant HIV-Infected Women for Maternal Health and Interventions to Reduce Perinatal HIV Transmission in the United States. Visitado en 2009 Sep 16. Disponible en http://aidsinfo.nih.gov/ contentfiles/Peri_Tables.pdf 
18. Committee on Obstetric Practice. ACOG committee opinion scheduled Cesarean delivery and the prevention of vertical transmission of HIV infection. Number 234, May 2000 (replaces number 219, August 1999). Int J Gynaecol Obstet 2001;73:279-81.

19. Shapiro D, Tuomala R, Samelson R, Burchett S, Ciupak G. McNamara J, et al. Mother-to-child HIV transmission rates according to antiretroviral therapy, mode of delivery, and viral load (PACTG 367). Abstract $114,9^{\text {th }}$ Conference on Retroviruses and Opportunistic Infections. 2002 Feb 24-28, Seattle, WA, U.S.A.

20. American College of Obstetricians and Gynecologists. ACOG practice bulletin number 47, October 2003: Prophylactic Antibiotics in Labor and Delivery. Obstet Gynecol 2003;102:875-82.

21. ACOG educational bulletin. Assessment of fetal lung maturity. Number 230, November 1996. Committee on Educational Bulletins of the American College of Obstetricians and Gynecologists. Int J Gynaecol Obstet 1997;56:191-8.

22. ACOG educational bulletin. Antimicrobial therapy for obstetric patients. Number 245, March 1998 (replaces no. 117, June 1988). American College of Obstetricians and Gynecologists. Int J Gynaecol Obstet, 1998;61:299-308.

23. Nielsen TF, Hokegard KH. Postoperative cesarean section morbidity: a prospective study. Am J Obstet Gynecol 1983;146:911-5.

24. Hebert PR, Reed G, Entman SS, Mitchel EF Jr, Berg C, Griffin MR. Serious maternal morbidity after childbirth: prolonged hospital stays and readmissions. Obstet Gynecol 1999;94:942-7.

25. Dunn DT, Newell ML, Ades AE \& Peckham CS. Risks of human immunodeficiency virus type 1 transmission through breast feeding. Lancet 1992;340:585-8.

26. Royal College of Obstetricians and Gynaecologists. Management of HIV in Pregnancy. Greentop Guideline No. 39, April 2004.

27. Centers for Disease Control and Prevention. Report of the NIH panel to define principles of therapy of HIV infection and guidelines for the use of antiretroviral agents in HIV-infected adults and adolescents. MMWR Morb Mortal Wkly Rep 1998;47:1-82.

28. Nduati R, John G, Mbori-Ngacha D, Richardson B, Overbaugh J, Mwatha A, et al. Effect of breastfeeding and formula feeding on transmission of HIV-1: a randomized clinical trial. JAMA 2000;283:1167-74.
29. Howie PW, Forsyth JS, Ogston SA, Clark A, Florey CD. Protective effect of breastfeeding against infection. BMJ 1990;300:11-6.

30. Young TK, Martens PJ, Taback SP, Sellers EA, Dean HJ, Cheang M, et al. Type 2 diabetes mellitus in children: prenatal and early infancy risk factors among native Canadians. Arch Pediatr Adolesc Med 2002;156:651-5.

31. Owen CG, Whipcup PH, Odoki K, Gilg JA, Cook DG. Infant feeding and blood cholesterol: a study in adolescents and systematic review. Pediatrics 2002;110:597-608.

32. Duncan B, Ey J, Holberg CJ, Wright AL, Martínez FD, Taussig LM. Exclusive breastfeeding for at least 4 months protects against otitis media. Pediatrics 1993;91:867-72.

33. UNAIDS. 2000 Report of the Global HIV/AIDS Epidemic. Visitado en 2009 Sep 16. Disponible en http:// www.aegis.com/files/unaids/WADDecember2000 epidemic_report.pdf

34. UNICEF. The State of the World's Children 1997. Visitado en 2009 Sep 16. Disponible en http://www. unicef.org/publications/files/pub_sowc97_en.pdf

35. Lawrence RA. Breastfeeding. A guide for the medical profession. $4^{\text {th }}$ ed. St Louis, MO: Mosby; 1994.

36. Lucas A, Morley R, Cole TJ Lister G, Leeson-Payne C. Breast milk and subsequent intelligence quotient in children born preterm. Lancet 1992;339:261-4.

37. Lanting CI, Fidler V, Huisman M, Touwen BC, Boersma ER, et al. Neurological differences between 9-year-old children fed breast-milk or formula-milk as babies. Lancet 1994;344:1319-22.

38. Fowler MG \& Newell ML. Breast-feeding and HIV-1 transmission in resource-limited settings. J Acquir Immune Defic Syndr 2002;30:230-9.

39. John-Stewart G, Mbori-Ngacha D, Ekpini R Janoff EN, Nkengasong J, Read JS, et al. Breast-feeding and transmission of HIV-1. J Acquir Immune Defic Syndr 2004;35:196-202.

40. Cutsoidis A. Breastfeeding and HIV. Best Pract Res Clin Obstet Gynaecol 2005;19:185-96.

41. American Academy of Pediatrics. Comittee on Pediatric AIDS. Evaluation and medical treatment of the HIVexposed infant. Pediatrics 1997;99:909-17.

42. British HIV Association. Guidelines for the management of HIV infection in pregnant women and the prevention of mother-to child transmission of HIV. Visitado en 2009 Sep 16. Disponible en: http://www.bhiva.org 
43. European Collaborative Study. Swiss mother, child HIV Cohort Study. Combination antiretroviral therapy, duration of pregnancy. AIDS 2002;14:1913-20.

44. Atkinson WL, Pickering LK, Schwartz B, Weniger BG, Iskander JK, Watson JC; Centers for Disease Control and Prevention. General Recommendations on Immunization. Recommendations of the Advisory Comittee on Immunization Practices (ACIP) and the American Academy of Family Physicians (AAFP). MMWR Recomm Rep 2002;51:1-35.

45. Recomendaciones de GESIDA/Plan Nacional sobre el Sida respecto al tratamiento antirretroviral en adultos infectados por el virus de la inmunodeficiencia humana (actualización enero de 2007); Panel de expertos de GESIDA y Plan Nacional sobre el Sida. Enferm Infecc Microbiol Clin 2007;25:32-53.

46. Panel on Clinical Practices for Treatment of HIV Infection convened by the U.S. Department of Health and Human Services (DHHS). Guidelines for the use of antiretroviral agents in HIV-infected adults and adolescents - Oct 10, 2006. Visitado en 2009 Sep 16. Disponible en: http://AIDSinfo.nih.gov
47. Gazzard B. British HIV Association (BHIVA) guidelines for the treatment of HIV-infected adults with antiretroviral therapy (2005). HIV Med 2005;6 Suppl 2:1-61.

48. Hammer SM, Saag MS, Schechter M, Montaner JS, Schooley RT, Jacobsen DM, et al. Treatment for adult HIV infection: 2006 recommendations of the International AIDS Society-USA panel. JAMA 2006;296:827-43.

49. Minkoff H, Augenbraun M. Antiretroviral therapy for pregnant women. Am J Obstet Gynecol 1997;176:478-89.

50. Lyons F, Hopkins S, Kelleher B, McGeary A, Sheehan G, Geoghegan, J, et al. Maternal hepatotoxicity with evirapine as part of combination antiretroviral therapy in pregnancy. HIV Med 2006;7:255-60.

51. Hitti J, Frenkel LM, Stek AM, Nachman SA, Baker D, Gonzalez-Garcia A, et al. Maternal toxicity with continuous nevirapine in pregnancy: results from PACTG 1022. J Acquir Immune Defic Syndr 2004;36:772-6. 